\title{
FORMULATION AND EVALUATION OF CHITOSAN CONTAINING MUCOADHESIVE BUCCAL PATCHES OF METOPROLOL SUCCINATE
}

\author{
Verma Navneet*, Verma Anurag, Dubey Juhi \\ Department of Pharmaceutics, Faculty of Pharmacy, IFTM University, Moradabad (UP) India \\ *Corresponding Author's Email: Navneet28jan@yahoo.com
}

Received 05 Feb 2016; Review Completed 28 Feb 2016; Accepted 05 March 2016, Available online 15 March 2016

\begin{abstract}
ABSTARCT
Mucoadhesive buccal patches containing metoprolol succinate were prepared using the solvent casting method. Chitosan was used as bioadhesive polymer and different ratios of chitosan to PVP K-30 were used. The patches were evaluated for their physical characteristics like mass variation, drug content uniformity, folding endurance, ex vivo mucoadhesion strength, ex vivo mucoadhesion time, surface $\mathrm{pH}$, in vitro drug release, and in vitro buccal permeation study. Patches exhibited controlled release for a period of $8 \mathrm{~h}$. The mechanism of drug release was found to be non-Fickian diffusion and followed the first-order kinetics. Incorporation of PVP K-30 generally enhanced the release rate. Swelling index was proportional to the concentration of PVP K-30. Optimized patches (F4) showed satisfactory bioadhesive strength of $9.6 \pm$ $2.0 \mathrm{~g}$, and ex vivo mucoadhesion time of 272 minutes. The surface $\mathrm{pH}$ of all patches was between 5.5 and 6.8 and hence patches should not cause irritation in the buccal cavity. Patches containing $10 \mathrm{mg}$ of drug had higher bioadhesive strength with sustained drug release as compared to patches containing $20 \mathrm{mg}$ of drug. Good correlation was observed between the in vitro drug release and in vitro drug permeation with a correlation coefficient of 0.9364 . Stability study of optimized patches was done in human saliva and it was found that both drug and buccal patches were stable.
\end{abstract}

Keywords: Chitosan, Poly-vinyl alcohol, Mucoadhesion, Buccal patch, Metoprolol succinate.

\section{INTRODUCTION}

Buccal mucosa is an attractive route for systemic delivery of drugs since it is relatively permeable with a rich blood supply. A drug can be easily applied and localized to the application site, and can be removed from there if necessary. Attempt has been made earlier to formulate various mucoadhesive buccal devices, including tablets, films, patches, disks, strips, ointments and gels. Buccal patches are highly flexible and thus much more readily tolerated by the patient than tablets. Patches also ensure more accurate dosing of the drug compared to gels and ointments.

During the last decade, bioadhesive polymers received considerable attention as plat-forms for buccal controlled delivery due to their ability to localize the dosage form in specific regions to enhance drug bioavailability. In the present study, the natural bioadhesive polymer chitosan was selected for the development of controlled release buccal mucoadhesive devices. Chitosan is the N-deacetylated product of the polysaccharide chitin . Chitosan is gaining increasing importance in the pharmaceutical field due to its good biocompatibility, after both intravenous and oral administration, and its nontoxicity and biodegradable. From the technological point of view, chitosan has also been demonstrated to be a promising matrix carrier for sustained drug release and it possesses excellent film-forming properties. In spite of this, only a few studies have so far been performed on the usefulness of chitosan films as drug delivery systems ${ }^{1}$.

Metoprolol succinate, a non-selective -adrenergic blocking agent, has been widely used in the treatment of hypertension, angina pectoris and many other cardio-vascular disorders ${ }^{2}$.

The present study was an attempt to develop chitosancontaining mucoadhesive buccal patches to ensure satisfactory release of metoprolol succinate for prolonged periods. The influence of Polyvinylpyrrolidone K-30 (PVP K-30) and drug concentration on the drug release and mucoadhesive performance on sheep buccal patches was investigated.

How to cite this article:

Verma N, Verma A, Dubey J, Formulation and evaluation of chitosan containing mucoadhesive buccal patches of metoprolol succinate, Journal of Drug Delivery \& Therapeutics. 2016; 6(2):1420 


\section{MATERIALS AND METHODS}

Metoprolol succinate $(99.00 \%$ purity) was a gift sample from Aarti drug Pvt Ltd., India. Chitosan (180 cps, $75 \%$ deacetylation) was procured from Sigma Aldrich, India. PVP K-30 (S. D. Fine Chemicals, India) was obtained from a commercial source. All other reagents and chemicals used were of analytical reagent grade.

\section{Preparation of mucoadhesive buccal patches}

Patches containing different drug and chitosan proportions were prepared by the solvent casting method. One gm of chitosan was dissolved in $100 \mathrm{ml}$ $1.5 \%(\mathrm{w} / \mathrm{v})$ acetic acid under occasional stirring for 24 hrs. The resulting viscous chitosan solution was filtered through whatman filter paper to remove suspended particles. To improve patch performance and release characteristics, a water-soluble hydrophilic additive, PVP K-30, was added in different concentrations. The drug and PVP K-30 were added into the chitosan solution under constant stirring. Propylene glycol $(10 \%$, v/v) was added into the solution as plasticizer under constant stirring. This viscous solution was left overnight at room temperature to ensure a clear, bubble-free solution. The solution was poured into a glass petri dish and allowed to dry at room temperature till a flexible film was formed. Dried films were carefully removed, checked for any imperfections or air bubbles and cut into patches of $16 \mathrm{~mm}$ in diameter, containing $20 \mathrm{mg}$ of drug per patch. The patches were packed in aluminum foil and stored in an airtight glass container to maintain the integrity and elasticity of the patches ${ }^{3}$. Table 1 shows the composition of different buccal patches.

Table 1: Composition of chitosan buccal patches of metoprolol Succinate

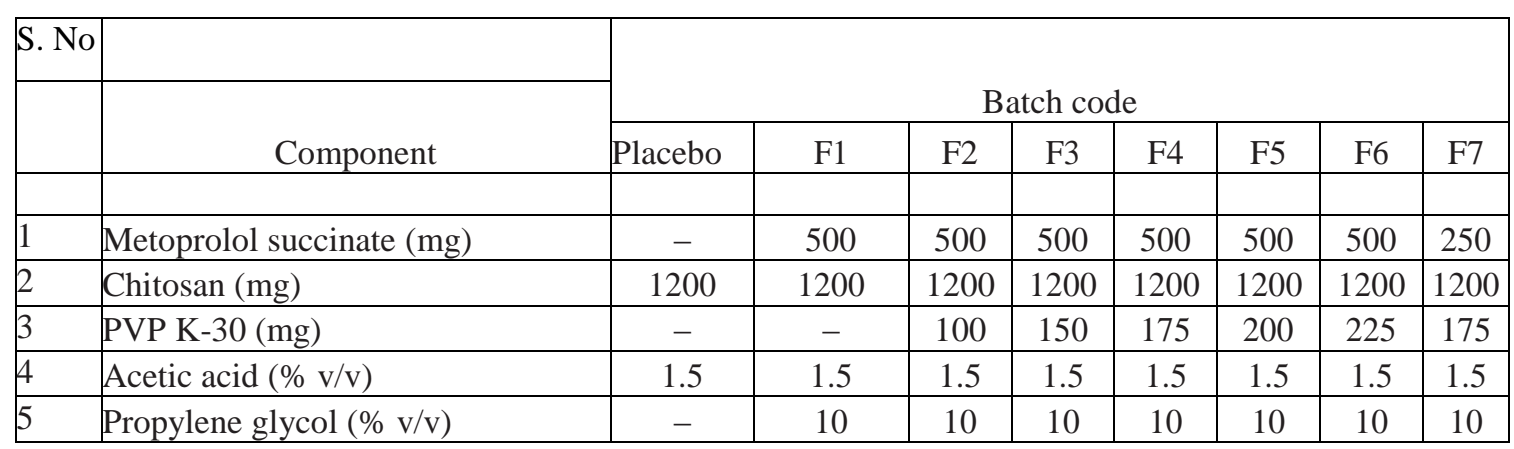

\section{Mass uniformity}

Mass uniformity was tested in 10 different randomly selected patches from each batch and patch thickness was measured at 5 different randomly selected spots using a screw gauge ${ }^{4}$.

\section{Folding endurance}

Folding endurance of the patches was determined by repeatedly folding one patch at the same place till it broke or folded up to 200 times without breaking 5 .

\section{Swelling study}

Buccal patch was weighed, placed in a $1.5 \%$ agar gel plate and incubated at $37 \pm 1{ }^{\circ} \mathrm{C}$. At regular one-hour time intervals up-to $3 \mathrm{~h}$, the patch was removed from the petri dish and excess surface water was removed carefully using the filter paper. The swollen patch was then reweighed and the swelling index was calculated. The experiments were carried out in triplicate and average values were reported ${ }^{6}$.

\section{Content uniformity}

Drug content uniformity was determined by dissolving the patch in $100 \mathrm{ml}$ of phosphate buffer $(\mathrm{pH}$ 6.8) for 8 $\mathrm{h}$ under occasional shaking. The $5 \mathrm{ml}$ solution was taken and diluted with isotonic phosphate buffer $\mathrm{pH}$ 6.8 up to $20 \mathrm{ml}$, and the resulting solution was filtered through a whatman filter paper. The drug content was then determined after proper dilution at $222 \mathrm{~nm}$ using a UV spectrophotometer (Shimadzu, 1800, Japan). The experiments were carried out in triplicate and average values were reported ${ }^{7}$.

\section{Ex-vivo mucoadhesive strength}

Fresh pig buccal mucosa was obtained from a local slaughterhouse and used within $2 \mathrm{~h}$ of slaughter. The mucosal membrane was separated by removing the underlying fat and loose tissues. The membrane was washed with distilled water at $37{ }^{\circ} \mathrm{C}$. Bioadhesive strength of the patch was measured $(n=3)$ on a modified physical balance. Fresh pig buccal mucosa was cut into pieces and washed with isotonic phosphate buffer $\mathrm{pH}$ 6.8. A piece of buccal mucosa was tied to the open mouth of a glass vial, filled completely with isotonic phosphate buffer $\mathrm{pH}$ 6.8. The glass vial was tightly fitted in the center of a glass beaker filled with isotonic phosphate buffer $(\mathrm{pH} 6.8$, $37 \pm 1{ }^{\circ} \mathrm{C}$ ). The patch was stuck to the lower side of the rubber stopper with cyanoacrylate adhesive. The weight, in gramms, required to detach the patch from the mucosal surface gave the measure of mucoadhesive strength $^{8,9}$. The following parameters were calculated from the bioadhesive strength: 
Bioadhesive strength $\times 9.8$

Force of adhesion $(\mathrm{N})=$ 1000

Force of adhesion

Bond strength $(\mathrm{N} \mathrm{m}-2)=\frac{}{\text { Surface area of petri dish }}$

The results are reported in Table 2

\section{Ex vivo mucoadhesion time}

The ex vivo mucoadhesion time was evaluated $(n=3)$ after application of the patches onto freshly cut pig buccal mucosa. The fresh pig buccal mucosa was fixed in the inner side of the beaker, above $2.0 \mathrm{~cm}$ from the bottom, with cynoacrylate glue. One side of each patch was wetted with one drop of isotonic phosphate buffer $\mathrm{pH} 6.8$ and pasted to the pig buccal mucosa by applying a light force with a fingertip for 30 seconds. The beaker was filled with $500 \mathrm{ml}$ of isotonic phosphate buffer $\mathrm{pH} 6.8$ and was kept at $37 \pm 1$ ${ }^{\circ} \mathrm{C}^{10,11,12}$. After 2 minutes, a $50 \mathrm{rpm}$ stirring rate was applied to simulate the buccal cavity environment, and patch adhesion was monitored up to $12 \mathrm{~h}$. The time required for the patch to detach from the pig buccal mucosa was recorded as the mucoadhesion time. The results are shown in Table 3.

Table 2: Bioadhesive parameters of metoprolol succinate buccal patches F1 to F7

\begin{tabular}{|c|c|c|r|}
\hline Batch code & Bioadhesive strength $(\mathrm{g})^{\mathrm{a}}$ & Force of adhesion $(\mathrm{N})$ & Bond strength $\left(\mathrm{N} \mathrm{m}^{-2}\right)$ \\
\hline Placebo & $20.4 \pm 1.5$ & 0.20 & 1034 \\
\hline F1 & $11.4 \pm 2.2$ & 0.11 & 611 \\
\hline F2 & $10.3 \pm 1.1$ & 0.11 & 549 \\
\hline F3 & $9.1 \pm 1.3$ & 0.10 & 491 \\
\hline F4 & $8.5 \pm 2.1$ & 0.09 & 464 \\
\hline F5 & $3.5 \pm 0.7$ & 0.04 & 231 \\
\hline F6 & $1.8 \pm 0.6$ & 0.02 & 136 \\
\hline F7 & $12.2 \pm 0.7$ & 0.11 & 647 \\
\hline
\end{tabular}

Table 3: Parameters of chitosan buccal patches of propranolol hydrochloride ${ }^{\mathrm{a}}$

\begin{tabular}{|c|c|c|c|c|c|c|}
\hline $\begin{array}{l}\text { Batch } \\
\text { Code }\end{array}$ & $\begin{array}{l}\text { Mass } \\
\qquad(\mathrm{mg})\end{array}$ & $\begin{array}{l}\text { Thickness } \\
\qquad(\mathrm{mm})\end{array}$ & $\begin{array}{l}\text { Drug } \\
\quad \text { content }(\%)\end{array}$ & $\begin{array}{l}\text { Ex vivo } \\
\text { mucoadhesion } \\
\text { time (min) }\end{array}$ & $\begin{array}{l}\text { Surface } \\
\\
\\
\mathrm{pH}\end{array}$ & $\begin{array}{l}\text { Folding } \\
\text { endurance }\end{array}$ \\
\hline Placebo & $47 \pm 1$ & $0.29 \pm 0.06$ & 0 & $703 \pm 4$ & $6.27 \pm 0.18$ & $206 \pm 15$ \\
\hline $\mathrm{F} 1$ & $71 \pm 1$ & $0.49 \pm 0.05$ & $99.15 \pm 0.30$ & $298 \pm 2$ & $5.81 \pm 0.16$ & $176 \pm 10$ \\
\hline $\mathrm{F} 2$ & $73 \pm 1$ & $0.52 \pm 0.02$ & $98.70 \pm 1.35$ & $281 \pm 4$ & $5.69 \pm 0.11$ & $175 \pm 10$ \\
\hline F3 & $75 \pm 1$ & $0.54 \pm 0.06$ & $100.00 \pm 0.40$ & $269 \pm 7$ & $6.00 \pm 0.12$ & $174 \pm 10$ \\
\hline F4 & $79 \pm 0$ & $0.55 \pm 0.01$ & $99.25 \pm 0.18$ & $271 \pm 4$ & $5.87 \pm 0.01$ & $162 \pm 15$ \\
\hline F5 & $81 \pm 1$ & $0.56 \pm 0.01$ & $99.68 \pm 0.14$ & $189 \pm 4$ & $5.77 \pm 0.11$ & $155 \pm 17$ \\
\hline F6 & $83 \pm 1$ & $0.57 \pm 0.02$ & $99.15 \pm 0.59$ & $161 \pm 2$ & $6.02 \pm 0.11$ & $149 \pm 20$ \\
\hline F7 & $67 \pm 1$ & $0.41 \pm 0.01$ & $99.78 \pm 0.22$ & $428 \pm 2$ & $5.82 \pm 0.03$ & $218 \pm 11$ \\
\hline
\end{tabular}

${ }^{\mathrm{a}}$ Mean $\pm \mathrm{SD}, \mathrm{n}=3$.

\section{Surface pH study}

A modified method adopted to determine the surface $\mathrm{pH}$ of the patches. A combined glass electrode was used for this purpose. The patches were allowed to swell by keeping them in contact with $1 \mathrm{ml}$ of distilled water for $2 \mathrm{~h}$ at room temperature, and $\mathrm{pH}$ was noted down by bringing the electrode in contact with the surface of the patch, allowing it to equilibrate for 1 minute $^{13}$.

\section{In vitro release}

The USP 23 rotating paddle method was used to study the drug release from buccal patches. The dissolution medium consisted of $200 \mathrm{ml}$ of isotonic phosphate buffer $\mathrm{pH}$ 6.8. The release was performed at $37 \pm 0.5$ ${ }^{\circ} \mathrm{C}$, at a rotation speed of $50 \mathrm{rpm}$. One side of the buccal patch was attached to a glass disk with cyanoacrylate. The disk was put in the bottom of the dissolution vessel so that the patch remained on the upper side of the disk. Samples $(2 \mathrm{ml})$ were withdrawn at pre-determined time intervals and replaced with fresh medium ${ }^{14,15}$. The samples were filtered through whatman filter paper with appropriate dilutions with phosphate buffer $\mathrm{pH} \quad 6.8$ and were assayed 
spectrophotometrically at $222 \mathrm{~nm}^{16}$.

\section{In vitro buccal permeation study}

The in vitro study of metoprolol succinate permeation through the pig buccal mucosa was performed using a diffusion cell at $37 \pm 0.5{ }^{\circ} \mathrm{C}$. Pig buccal mucosa was obtained from a local slaughterhouse. Freshly obtained pig buccal mucosa was mounted between the donor and receptor compartments so that the smooth surface of the mucosa faced the donor compartment ${ }^{17}$. The patch was placed on the mucosa and the compartments clamped together. The donor compartment was filled with $1 \mathrm{ml}$ of isotonic phosphate buffer $\mathrm{pH}$ 6.8. The receptor compartment ( $25 \mathrm{ml}$ capacity) was filled with isotonic phosphate buffer $\mathrm{pH} 7.4$ and the hydrodynamics in the receptor compartment was maintained by stirring with a magnetic bead at $50 \mathrm{rpm}$. One $\mathrm{ml}$ sample was withdrawn at predetermined time intervals and analyzed for drug content at $222 \mathrm{~nm}$.

\section{Stability in phosphate buffer}

The stability of optimized patches was tested in isotonic phosphate buffer ( $\mathrm{pH}$ 6.8). Patches were placed in separate Petri dishes containing $5 \mathrm{ml}$ of phosphate buffer and kept in a temperature-controlled oven at $37 \pm 0.2{ }^{\circ} \mathrm{C}$ for $6 h^{18,19}$. At regular time intervals $(0,1,2,3$ and $6 \mathrm{~h})$, the patches were examined for changes in color and shape, collapse of the patch. Drug content was determined by appropriate dilution and analyzed by spectrophotometry at $222 \mathrm{~nm}$.

\section{RESULTS AND DISCUSSION}

In the present study, buccal patches for controlled delivery of metoprolol succinate were developed using chitosan as the base matrix. The patches were prepared using different ratios of chitosan to PVP K-30 from batch F1 to F6. PVP K-30 was added to improve the drug release by polymer swelling, elasticity and film forming properties of the patches. Propylene glycol $(10 \%)$ was added as plasticizer. On the basis of bioadhesive strength $(8.5 \pm 2.1 \mathrm{~g})$ and in vitro drug release $(82.2 \%$ in $7 \mathrm{~h}$ ) from the buccal patches, batch F4 was selected for further study. Batch F7 was prepared from optimized batch (F4) by taking half quantity of the drug to study the effect of drug concentration in buccal formulations.

The prepared patches were smooth in appearance, uniform in thickness, mass, and drug content and showed no visible cracks. The patches exhibited good folding endurance (more than 150, Table 3). Patch thickness ranged from $0.41 \pm 0.01$ to $0.57 \pm 0.02 \mathrm{~mm}$ and mass ranged from $67 \pm 1$ to $83 \pm 1 \mathrm{mg}$. Patches had a surface $\mathrm{pH}$ of $5.69 \pm 0.11$ to $6.02 \pm 0.11$. The drug content in the buccal patches ranged from $98.7 \pm$ 1.3 to $100.0 \pm 0.4 \%$, indicating the favourable drug loading and patches uniformity with respect to drug content.

Appropriate swelling behavior of a buccal adhesive system is the essential property for uniform and prolonged release of the drug and effective mucoadhesion. The swelling study indicated that the swelling index was higher in patches containing a higher amount of PVP K-30. Examination of the patches during the dissolution studies also revealed that the patches showed considerable swelling, especially at higher concentrations of PVP K-30. The weak aqueous solubility of the cationic polymer (chitosan) limited the swelling of the patches, which was observed in placebo patches. Addition of the hydrophilic polymer PVP K-30 increased the surface wettability and consequently water penetration within the matrix.

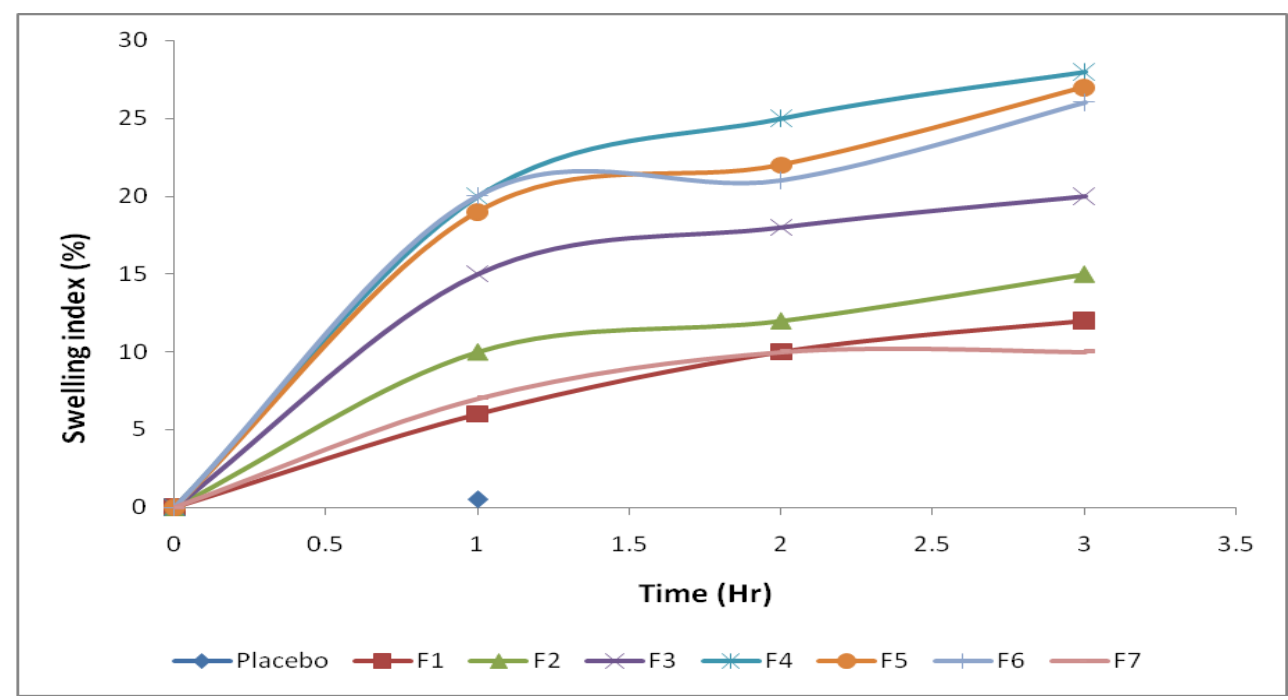

Figure 1: Swelling index of buccal patches from batches F1 to F7. Mean $\pm \mathrm{SD}, n=3$.

Patches did not show any appreciable changes in their shape and form during the $3 \mathrm{~h}$ that they were kept on a $1.5 \%$ agar gel plate. The optimized patch $(\mathrm{F} 4)$ showed a $28.8 \pm 0.8 \%$ swelling index due to water absorption within $3 \mathrm{~h}$. Swelling behavior of patches as a function of time is shown in Figure 1. It was observed that medicated patches had a higher swelling index compared to plain patches. The higher swelling index 
of medicated patches may be due to the hydrophilic nature of the drug, which dissolves fast when it comes in contact with the dissolution medium.

Mucoadhesion may be defined as the adhesion between a polymer and mucus. In general, mucoadhesion is considered to occur in 3 major stages: wetting, interpenetration, and mechanical interlocking between mucus and polymer. The strength of mucoadhesion is affected by various factors such as molecular mass of polymers, contact time with mucus, swelling rate of the polymer and the biological membrane used in the study. In this study, pig buccal mucosa was used as biological membrane. Plain patches showed higher mucoadhesive strength $(20.4 \pm$ $1.5 \mathrm{~g}$ ) than medicated patches. The patch containing 10 mg of drug (F7) showed higher bioadhesive strength compared to $20 \mathrm{mg}$ of drug (F1 to F6). Incorporation of PVP K-30, a water-soluble hydrophilic polymer, and water-soluble drug reduced significantly the bioadhesive strength of buccal patches. Bioadhesive strength of the optimized patch $(\mathrm{F} 4)$ was found to be $8.5 \pm 2.1 \mathrm{~g}$ and the force of adhesion and bond strength were $0.0932 \mathrm{~N}$ and $464.82 \mathrm{~N} \mathrm{~m}^{-2}$, respectively. The important bioadhesive parameters of buccal patches are given in Table 2.

In vitro release of metoprolol succinate from different patches is shown in Figure 2. The drug released increased linearly with the increasing concentration of PVP-K-30 from batches F1 to F6. The maximum in vitro release was found to be $99.2 \pm 0.7 \%$ over a period of $7 \mathrm{~h}$ in batch F6, containing the highest amount of PVP K-30, which could be attributed to its high rate and extent of swelling. This finding was also supported by the results of swelling studies where the highest swelling index was also exhibited by batch F6, indicating that the increase in water-soluble polymer PVP-K30 content results in faster swelling and release from patches.

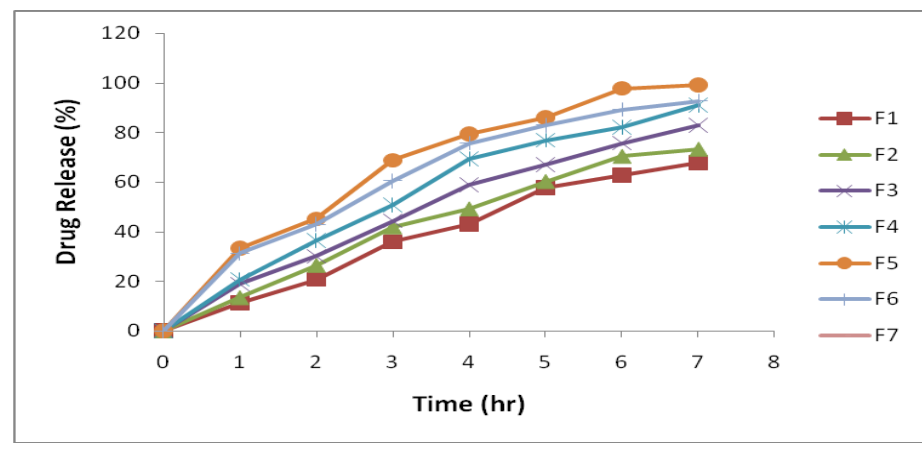

Figure 2: Cumulative drug released from batches F1 to F7. Mean $\pm \mathrm{SD}, n=3$.

F4 patches with $20 \mathrm{mg}$ of drug showed higher and faster drug release than patches containing $10 \mathrm{mg}$ of drug (F7). metoprolol succinate (a water soluble drug), dissolves easily in a hydrated polymeric environment. Therefore, the higher the loading of metoprolol succinate, the more drug would dissolve inside the hydrated matrices, resulting in a higher diffusional driving force and faster drug release. These results showed that the drug and PVP K-30 have also a significant effect on release behaviour of the drug from chitosan-based matrix. The release data were analyzed using the well known semi-empirical Peppas equation:

$$
\mathrm{M}_{\mathrm{t}} / \mathrm{M}_{¥}=\mathrm{k} \mathrm{t}^{\mathrm{n}}
$$

where $M_{t} / M_{¥}$ is the fractional release of the drug, $t$ denotes the release time, $\mathrm{k}$ represents a kinetic constant, incorporating structural and geometrical characteristics of the device, and $\mathrm{n}$ is the diffusional exponent and characterizes the type of release mechanism during the dissolution process. For nonFickian release, the value of $\mathrm{n}$ falls between 0.5 and 1.0 while in the case of Fickian diffusion $n=0.5$; for first order release (case II transport) $\mathrm{n}=1$, and for supercase II transport $\mathrm{n}>1$. The obtained values of $\mathrm{k}, \mathrm{n}$ and $\mathrm{R}^{2}$ (coefficient determination) are presented in Table 4.

Table 4: Kinetic constant (k), release exponent (n) and determination coefficient $\mathrm{R}^{2}$

\begin{tabular}{|c|c|l|l|}
\hline \multirow{2}{*}{ Batch } & & Peppas model & \\
\cline { 2 - 4 } & $\mathrm{k}\left(\% \mathrm{~h}^{-1}\right)$ & $\mathrm{R}^{2}$ & $\mathrm{n}$ \\
\hline F1 & 0.1611 & 0.9674 & 0.655 \\
\hline F2 & 0.1817 & 0.9869 & 0.673 \\
\hline F3 & 0.1875 & 0.9914 & 0.7025 \\
\hline F4 & 0.2011 & 0.9926 & 0.7354 \\
\hline F5 & 0.2102 & 0.9941 & 0.7622 \\
\hline F6 & 0.2152 & 0.9916 & 0.7810 \\
\hline F7 & 0.1830 & 0.9886 & 0.6931 \\
\hline
\end{tabular}




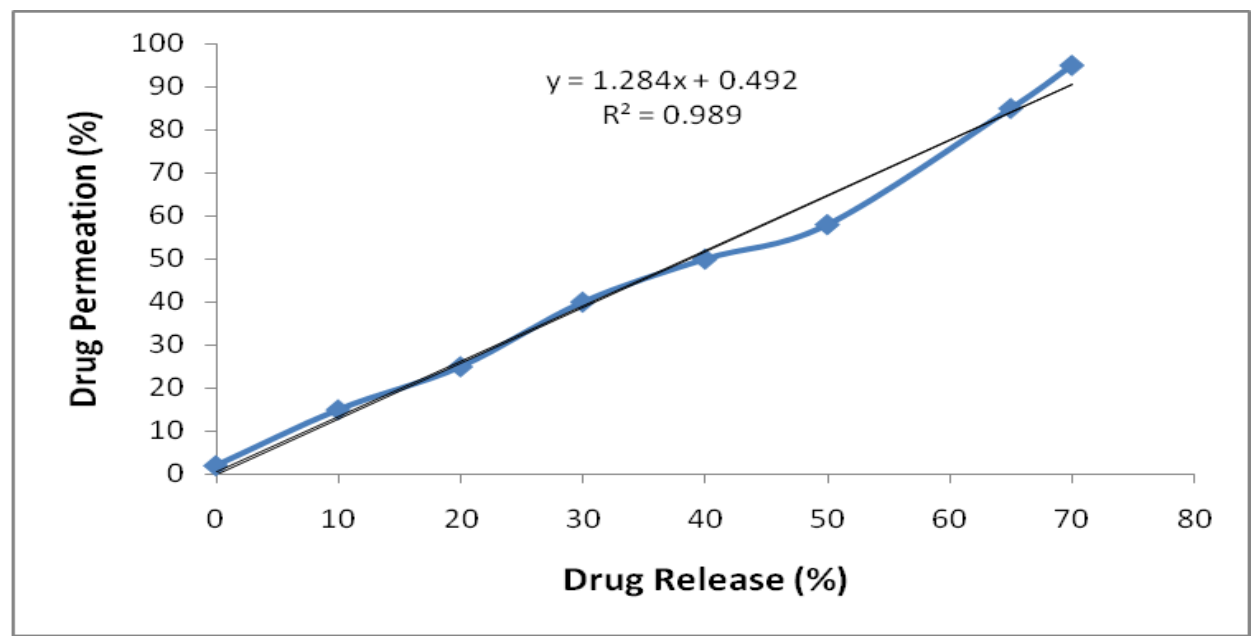

Figure 3: Correlation between in vitro drug release and in vitro drug permeation.

The values of $\mathrm{n}$ were estimated by linear regression of $\log \left(\mathrm{M}_{\mathrm{t}} / \mathrm{M}\right)$ vs. $\log \mathrm{t}$, and were between 0.5 and 1.0, indicating that the release of metoprolol succinate was by non-Fickian diffusion. In the kinetics study, the order of drug release from all batches followed zero-order kinetics.

The surface $\mathrm{pH}$ of the patches was determined in order to investigate the possibility of any side effects, in vivo. Since an acidic or alkaline $\mathrm{pH}$ may cause irritation to the buccal mucosa, we attempted to keep the surface $\mathrm{pH}$ as close to neutral as possible. The surface $\mathrm{pH}$ of all the patches (F1 to F7) was near 7 and hence, these patches should not cause any irritation in the buccal cavity. Ex vivo mucoadhesion time for the medicated patches varied from 161 to 428 minutes (Table 3, the plain patches showed longer mucoadhesion time (703 minutes). Incorporation of PVP K-30 and the drug reduced significantly ex vivo mucoadhesion time of the patches. Optimized patches (F4) showed a 271 minutes mucoadhesion time on sheep buccal mucosa.

F4 patches were characterized by moderate swelling, a convenient residence time as well as adequate drug release. These patches were subject to investigation of in vitro drug diffusion and stability in phosphate buffer. They showed $82.9 \%$ drug permeation in $7 \mathrm{~h}$ through pig buccal mucosa. Good correlation was observed between in vitro drug release and in vitro drug permeation with a correlation coefficient of 0.98 (Figure. 3).

Table 5: Stability study of optimized chitosan buccal patches (batch F4) in phosphate buffer

\begin{tabular}{|c|c|c|c|}
\hline Sampling time $(\mathrm{h})$ & ${\text { Thickness }(\mathrm{mm})^{\mathrm{a}}}^{\mathrm{a}}$ & ${\text { Diameter }(\mathrm{mm})^{\mathrm{a}}}$ & ${\text { Drug recovered }(\%)^{\mathrm{a}}}$ \\
\hline 0 & $0.55 \pm 0.05$ & $15.9 \pm 0.11$ & $99.7 \pm 0.2$ \\
\hline 1 & $0.55 \pm 0.07$ & $16.0 \pm 0.10$ & $99.0 \pm 0.4$ \\
\hline 2 & $0.56 \pm 0.06$ & $16.1 \pm 0.03$ & $99.4 \pm 0.4$ \\
\hline 3 & $0.59 \pm 0.01$ & $16.2 \pm 0.02$ & $99.6 \pm 0.2$ \\
\hline 6 & $0.59 \pm 0.03$ & $16.4 \pm 0.09$ & $99.3 \pm 0.3$ \\
\hline
\end{tabular}

${ }^{\mathrm{a}}$ Mean $\pm \mathrm{SD}, \mathrm{n}=3$.

Stability studies are performed in phosphate buffer solutions whose $\mathrm{pH}$ pertains to the buccal cavity. The stability study of optimized patches (F4) was examined in phosphate buffer and their appearance characteristics, such as color and shape, and drug content in phosphate buffer saliva were evaluated (Table 5). Thickness and diameter of patches increased to 7.3 and $3.4 \%$ owing to swelling in phosphate buffer in $6 \mathrm{~h}$ studies. No color changes were observed. The recovery of the drug from all patches was found to be $99.7 \pm 0.2 \%$ indicating maximum utilization of the drug incorporated.

\section{CONCLUSIONS}

From the present investigation, one can conclude that the optimized buccoadhesive patches metoprolol succinate with the combination of chitosan and PVP K-30 can meet the ideal requirements for buccal devices, which can be a good way to bypass the extensive hepatic first pass metabolism. 


\section{REFERENCES}

1. Khairnar A, Parridhi $\mathrm{J}$ and Dheeraj B, Development of Mucoadhesive Buccal patch containing Aceclofenac : In vitro evaluations, Int. J. Pharm. Tech Res., 2009, 1, 978-981.

2. Randhawa MA, Malik S A and Javed M Buccal absorption of weak acidic drugs is not related to their degree of ionization as estimated from the Henderson-Hasselbalch equation, Pakistan J. Med. Res., 2003, 42, 116-119.

3. Consuelo ID and Francoise F, Ex vivo evaluation of bioadhesive films for buccal delivery of fentanyl, J. Cont. Rel, 2007, 122, 135-140.

4. Kokate AA and Xiaoling L, HPLC detection of marker compounds during Buccal permeation enhancement studies, J. Pharm. Biomed. Anal., 2008, 47, 190-194.

5. Hao J and Heng PWS, Buccal Delivery System, Drug Develop. Indus. Pharmacy,2003, 29, 821-832.

6. Scholz O A and Wolff A, Drug delivery from the oral cavity: focus on a novel mechatronic delivery device, Drug discovery today, 2008, 13, 247-253.

7. Nafee N A and Ismail F A, Mucoadhesive delivery system. II. Formulation and In-vitro/In-vivo evaluation of buccal mucoadhesive tablets containing water soluble drugs, Develop. Indus. Pharm., 2004, 30, 995-1004.

8. Riikka L, Eera S, Kaisa T and Mikko B, Intraorally fast dissolving particles of a poorly soluble drug: Preparation and in vitro characterization, Eur. J. Pharm. Biopharm., 2009, 71, 271-281.

9. Aleksandra MS and Kijenska E, Electro-osmotic flow as a result of Buccal iontophoresis- Buccal mucosa properties, Eur. J. Pharm. Biopharm., 2009, 72, 595-599.

10. Perioli L and Ambrogi V, Development of mucoadhesive patches for buccal administration of Ibuprofen, J. Cont. Rel., 2004, 99, 73-82.
11. Cafaggi $\mathrm{S}$ and Leardi $\mathrm{R}$, Preparation and evaluation of a chitosan salt-poloxamer 407 based matrix for buccal drug delivery, J. Cont. Rel., 2005, 102, 159-169.

12. Lehr CM, Bouwstra JA, In vitro evaluation of mucoadhesive properties of chitosan and some other natural polymer. Int. J. Pharmaceutics., 1992; 78, 43-8.

13. Shojaei $\mathrm{AH}$ and $\mathrm{Li} \mathrm{X}$, Determination of transport route of acyclovir across buccal mucosa, Cont. Rel. Bioactive Mat., 1997, 24, 427-438.

14. Ana F, Juliane $\mathrm{H}$ and Andreas BS, In-vitro evaluation of natural and methylated cyclodextrin as Buccal permeation enhancing system for omeprazole delivery, Eur. J. Pharm. Biopharm., 2009, 71 339-345.

15. Ellen $\mathrm{H}$, Marianne $\mathrm{H}$ and Arne $\mathrm{S}$, Mucoadhesive and drug permeation of free mixed films of pectin and Chitosan : An in vivo study, Eur. J. Pharm. Biopharm, 2009, 71, 325-331.

16. Albertini B, and Passerini M, Polymer-lipid Based mucoadhesive microspheres prepared by spray-congealing for the vaginal delivery of econazole nitrate, Eur. J. Pharm., 2009, 36, 591-601.

17. Henrihsen I and Green LK, Bioadhesion of hydrated chitosan : an in vitro and in vivo study, Int. J. Pharmaceutics, 1996, 145, 231-240.

18. Vishnu MP and Bhupendra GP, Design and characterization of Chitosan containing Mucoadhesive Buccal patches of propanolol HCl, AAPS Pharma. Sci. Tech, 2007, 67, 61-72.

19. Mashru RC and Sutariya MG, Development and evaluation of fast dissolving film of salbutamol sulphat, Drug develop. Ind. Pharm., 2005, 1, 25-34. 